\title{
The prevalence of HIV among patients admitted with stroke at the Muhimbili National Hospital, Dar es Salaam, Tanzania
}

\author{
M. MLAY and M. BAKARI* \\ Department of Internal Medicine, Muhimbili University of Health and Allied Sciences, \\ P.O. Box 65001, Dar es Salaam, Tanzania
}

\begin{abstract}
Stroke and HIV infection are both common medical problems in the day to day clinical practice. Although data from developed countries confirm HIV infection as a risk for stroke the exact underlying mechanism is still unclear. Little data exist on the magnitude of HIV among patients with stroke in Tanzania. This cross-sectional study was carried out to determine the prevalence of HIV and assess its impact on the clinical presentation and outcome of patients admitted with stroke at the Muhimbili National Hospital, Dar es Salaam, Tanzania between May and November 2008. Patients were clinically evaluated through history and physical examination. Presence of HIV was determined by demonstration of HIV antibodies through an ELISA test. CD4 ${ }^{+}$T-lymphocyte count was determined by flowcytometry. Complete blood counts, as well as lipid profile and blood glucose levels were also determined. A total of 215 patients with stroke, constituting $6.8 \%$ of the admissions, were analyzed. The prevalence of HIV among patients with stroke was $20.9 \%(45 / 215)$. The overall mean $( \pm S D)$ age of patients with stroke who were HIV infected was $47.2( \pm 14.5)$ years, while that among patients with stroke but HIV un-infected was $56.1( \pm 15.1)$ years $(P<0.001)$. The proportion of patients with stroke and HIV who had hypertension was 53.3\% (24/45), whereas among those HIV uninfected was $80.6 \%(137 / 170)(P=<0.001)$. Furthermore, fever, anaemia, diarrhoea, tuberculosis and Kaposi's sarcoma were significantly more prevalent among those with HIV than those with no HIV infection. The respective proportions were $44 \%$ vs $24.7 \% ; 26.7 \%$ vs $7.6 \% ; 20.0 \%$ vs $2.9 \% ; 13.3 \%$ vs $1.2 \%$; and $6.7 \%$ vs $0 \%(P<0.01)$. Majority $(58.3 \%)$ of the HIV infected stroke patients had CD4 counts of less than 200cells/ $\mu \mathrm{l}$. The mean duration of hospital stay of 10.3 days was significantly longer among those with HIV compared to that of 7.3 days among the HIV un-infected patients with stroke $(P=0.001)$. While on univariate analysis both fever and anaemia were associated with mortality, on multivariate analysis the presence of fever was found to be significantly associated with mortality among patients with stroke and HIV. In conclusion, the prevalence of HIV infection among patients with stroke was high. Patients with stroke and HIV were younger, had significant immunodeficiency and presented with other HIV-related illnesses. Early detection of HIV through enhanced counselling and testing is recommended.
\end{abstract}

Key words: HIV, stroke, prevalence, hospital, Tanzania

\section{Introduction}

Cardiovascular diseases including stroke are predicted to overtake infectious diseases as a cause of morbidity and mortality in Sub-Saharan African countries by the year 2020 (Murray, 1997; Yusuf, 2004), largely as a result of changing life style associated with urban life (Connor, 2004). Furthermore, it is also known that among blacks, stroke is more common, more severe, and carries a higher mortality when compared with other races. This is attributed by the fact that blacks have an increased frequency of traditional risk factors for stroke such as diabetes mellitus, hypertension, and obesity (Jones \& Smith-Hammond, 2000; Walker, 2000; Imam, 2002).

\footnotetext{
* Correspondence: Dr. Muhammad Bakari; E-mail: drbakari@yahoo.com; mbakari@muhas.ac.tz
} 
Although stroke is more common in the elderly, in $25 \%$ they do occur in young adults in whom HIV may be the major risk factor (Hoffmann, 2000; Imam, 2002). In a retrospective study among 1,600 patients with HIV/AIDS, 12 cases of strokes $(0.75 \%)$ were recorded in five years (Engestrom et al., 1989). Comparing this number with the annual incidence of stroke among young adults (35-45 years) in the general population of $0.025 \%$, the authors concluded that patients with AIDS seem to be at a higher risk for stroke. Another retrospective study done in USA found HIV to be associated with an increased risk of stroke, particularly cerebral infarction in young adults (Qureshi et al.,, 1997). Furthermore, a study conducted in the medical ward of Queen Elizabeth Central Hospital in Blantyre, Malawi found that among 98 patients with stroke, 48\% had HIV infection (Kumwenda et al.,, 2005).

Although these and other studies (Evers et al., , 2003; Cole et al., 2004) appear to confirm that HIV infection is a risk factor for stroke, the exact underlying mechanism is still unclear. It is proposed that since HIV may cause dilated cardiomyopathy and predispose to meningitis, this would lead to an increased prevalence of well established risk factors such as cardiac embolism and meningitis (Cosnett, 1969; Bahemuka, 1979; Kumwenda et al., 2005). It is reported in literature that in about half of patients with AIDS, one can find a presence of transient neurological deficit or cerebral infarction (Brew, 1993). The common causes of cerebral infarction in these patients are non-bacterial thrombotic endocarditis and opportunistic central nervous infections like toxoplasmosis, cryptococcal meningitis and tuberculosis (Brew, 1993). Others have suggested that HIV patients have elevated systemic inflammation making them prone to atherosclerosis (Stollberger \& Finsterer, 2002).

It is also thought that HIV can induce vascular damage resulting into a coagulopathy by induction of autoantibodies and protein S deficiency (Mochan et al., 2005). The contribution of toxic effects of viral antigen on vasculopathy has also been described, especially in relation to HIV infected patients with low CD 4 cell counts who are reported as well to have a higher risk for coronary events through atherosclerosis. In one study aimed at identifying the predictors of carotid intima-media thickness in HIV patients, the thickness was found to be higher in HIV patients than their age-matched controls and progressed much more rapidly than previously reported rates in non-HIV cohorts. The carotid intimamedia thickness was associated with the classic coronary risk factors, with a nadir CD4 ${ }^{+}$ count of $\leq 200$, suggesting that immunodeficiency and traditional risk factors may contribute to atherosclerosis (Hsue et al., 2004).

The use of highly active antiretroviral therapy (HAART) has also been reported to be associated with several metabolic complications that include hyperlipidemias that are established risk factors for cerebrovascular events including stroke (Brown et al., 2005). This is especially so with the use of protease inhibitors which are also recommended as second line drugs for the treatment of HIV infection in Tanzania. The use of Lopinavir/Rotonavir has been reported to be associated with a mean increase in total serum cholesterol of $49 \mathrm{mg} / \mathrm{dl}$; and that of triglycerides of $111 \mathrm{mg} / \mathrm{dl}$ at 48 weeks of follow-up (Murphy et al., 2001).

The delineation of commoner stroke type between infarction and haemorrhage is a challenge in resource-poor countries like Tanzania where imaging techniques such as CT scan and MRI are very limited. Available literature suggests that the majority of strokes are ischemic in type, and its prevalence could be increasing. However, whereas among South African blacks the proportion of ischaemic strokes was estimated at 71.2\% (Joubert, 1991), a study in Tanzania found that among 89 patients with stroke, the CT scan revealed that $60.9 \%$ had hemorrhagic type of stroke (Matuja 2004). However, it is generally thought that age has 
an impact on this with hemorrhagic strokes being commoner among the younger ages (Wiredu \& Nyame, 2001). On the other hand, it would appear that the prevalence of stroke is not significantly different between the male and female gender (Stuart-Shor et al., 2009).

HIV and AIDS continue to be a major public health problem in Tanzania. The reported national HIV prevalence is $6.0 \%$. The prevalence among women is $7.0 \%$, while that among men is $5.0 \%$. The $30-39$ years age group is predominantly affected (TACAIDS, 2008). It is therefore important to study the influence of HIV on the pattern of strokes as seen in Tanzania so as to better inform the subsequent management. The objective of this study was to determine the prevalence of HIV and assess its impact on the clinical presentation and outcome of patients admitted with stroke at the Muhimbili National Hospital, Dar es Salaam, Tanzania.

\section{Materials and Methods}

\section{Study site and subjects}

This was a descriptive cross sectional study conducted between May and November 2008 among patients admitted with stroke in the Medical wards of the Muhimbili National Hospital in Dar es Salaam. This is the largest and only tertiary referral hospital in Tanzania, with patients being referred from both public and private health facilities as well as from neighbouring regions.

The sample size was calculated by adopting the HIV prevalence of $48 \%$ among patients with stroke in a study done in Malawi (Kumwenda et al., 2005). A maximum likely error of $7 \%$ with significance levels of $5 \%$ was used. An allowance of $10 \%$ was entertained for possible non-response. All admitted patients were clinically examined to elicit symptoms and signs of stroke and HIV and any other disease. Those with stroke were included in the study. Blood pressure was measured with a mercury sphygmomanometer on the left arm, two times at 5 minutes intervals. The $1^{\text {st }}$ and the $5^{\text {th }}$ Korotokoff's sounds were used to determine the systolic (SBP) and diastolic blood pressure (DBP) measurements respectively. The second blood pressure measurement was used as the blood pressure for the individual. Weight was measured using a weighing machine and recorded to the nearest $0.5 \mathrm{~kg}$. Height was measured using a tape measure to the nearest centimetres. The waist circumference was measured using a tape measure.

A Siriraj stroke score was used to determine the stroke subtype. The score is based on clinical parameters and has been shown to have good predictive value (Poungvarin et al., 1991; Nyandaiti \& Bwala, 2008). The score includes (2.5x level of consciousness) $+(2 x$ vomiting $)+(2 x$ headache $)+(0.1 x$ diastolic blood pressure $)-(3 x$ atheroma markers $)-12$. A score above 1 indicates cerebral haemorrhage, score below -1 infarction, and between 1 and 1 represents an equivocal situation. However, because the score can exclude haemorrhage more than infarction then those who fall between 1 and -1 are most likely to be having infarction (Murphy et al., 2001).

Clinical variables were scored as follows; level of consciousness was scored as alert ( 0 point), drowsy or stuporous (1 point), semi-comatose or comatose ( 2 points). Headache as well as, atheroma markers defined as history of diabetes, hypertension in the past, and presence of heart problem were each scored as 1 point (Poungvarin et al., 1991). The information obtained was filled in a structured questionnaire and later on entered into a computer. 


\section{Laboratory investigations}

For laboratory investigations, $5 \mathrm{ml}$ of venous blood was taken from each individual. HIV testing was performed using ELISA test for HIV antibodies. Blood samples that were not reactive were considered HIV negative. For all reactive blood samples, a second ELISA was performed to confirm the initial result. HIV positivity was entertained when both tests reacted. In an event where blood sample was indeterminate, a confirmatory test using Western Blot (WB) was performed. Determination of CD4+ T-lymphocytes counts was done using Facs count machine at the Department of Microbiology and Immunology of Muhimbili University of Health and Allied Sciences in Dar es Salaam. Determination of lipid profile, blood glucose levels and hemoglobin were done at the Muhimbili Orthopaedic Institute laboratory. The lipid profile was tested using Vitros DT 6011 Chemistry Analyzer. Haemoglobin levels were determined using HEMOCUE HB 201+. Blood glucose level was done on the spot after collection using HEMOCUE Glucose 201+.

\section{Definition of terms}

Stroke patient was defined as any patient presenting with features of acute neurological deficit in the absence of a history of trauma (Kasper et al., 2005). Diabetes mellitus was defined as a Fasting Blood Glucose of more that $7.0 \mathrm{mmol} / \mathrm{L}$ or a Random Blood Glucose of more than $11.1 \mathrm{mmol} / \mathrm{l}$ (Nicholas et al., 2006). Hypertension was defined as a Systolic Blood Pressure (SBP) $\geq 140$ and/or a Diastolic Blood Pressure (DBP) of $\geq 90 \mathrm{mmHg}$ or the use of hypertensive medications prior to onset of stroke. Obesity was defined as waist circumference $\geq 88 \mathrm{cms}$ in women, $\geq 102 \mathrm{~cm}$ in men (Erem et al., 2004). Immunodeficiency was defined as an absolute $\mathrm{CD} 4{ }^{+}$cell count of less than 200 cells $/ \mu 1$. Anaemia was defined as a haemoglobin level of less than 13 and $12 \mathrm{~g} / \mathrm{dl}$ in men and women respectively. Fever was defined as a body temperature equal to or above $37.5^{\circ} \mathrm{C}$.

\section{Ethical consideration}

Information about the study was explained to patients themselves, or to relatives or guardians for those who were unconscious or mentally incapable of giving informed consent prior to recruitment. There was none among the eligible patients whose consent could not be obtained. Ethical clearance for the study was obtained from the Muhimbili University of Health and Allied Sciences, Senate Research and Publications Committee. All newly diagnosed patients with HIV-infection were referred to the HIV care and treatment clinic for further management.

\section{Data analysis}

Data was analysed using a Statistical Package for Social Sciences (SPSS) version 10.5. Comparison of proportions was done using the Chi-squared test, and Fisher's exact test was adopted when the expected value in a cell was less than five. Means were compared using Student's $t$-test. Risk factors for mortality were assessed through Logistic regression. Statistical significance for differences was considered if the p-value was less than or equal to 0.05 . 


\section{Results}

\section{Stroke and HIV infection}

During the study period 3,255 patients were admitted in the Medical wards, of whom 220 $(6.8 \%)$ had stroke. Of the 220 patients with stroke, 51.4\% were males. Five of the patients with stroke had in-determinate HIV results both on ELISA and Western blot tests. These were excluded from further analysis. The overall prevalence of HIV infection among patients presenting with stroke was $20.9 \%$ (45/215). The proportion of female patients with stroke did not differ by HIV serostatus, being 55.6\% among those with HIV-infection and $46.5 \%$ among those who were HIV negative $(P=0.18)$. Similarly, there were no differences in the proportion of male patients with stroke by HIV serostatus (Table 1).

Table 1: Social Demographic Characteristics of patients presenting with stroke by their HIV status

\begin{tabular}{|c|c|c|c|c|}
\hline \multirow[t]{2}{*}{ Characteristic } & \multirow{2}{*}{ Variable } & \multicolumn{2}{|c|}{ HIV status } & \multirow{2}{*}{ P-value } \\
\hline & & Positive, $n=45$ & Negative, $n=170$ & \\
\hline \multirow[t]{2}{*}{ Sex, n (\%) } & Male & $20(44.4 \%)$ & $91(53.5 \%)$ & 0.18 \\
\hline & Female & $25(55.6 \%)$ & 79 (46.5\%) & \\
\hline \multirow[t]{3}{*}{ Mean age (SD) } & All & $47.2(14.5)$ & $56.1(15.1)$ & $<0.001$ \\
\hline & Male & $46.3(14.5)$ & $56.6(14.4)$ & \\
\hline & Female & $47.9(14.8)$ & 55.5 (17.4) & \\
\hline \multirow[t]{6}{*}{ Age distribution, n (\%) } & $<25$ & $3(6.7 \%)$ & $6(3.5 \%)$ & 0.18 \\
\hline & $25-34$ & $5(11.1 \%)$ & $10(5.9 \%)$ & \\
\hline & $35-44$ & $8(17.8 \%)$ & 17 (10.0\%) & \\
\hline & $45-54$ & $13(28.9 \%)$ & $50(29.4 \%)$ & \\
\hline & $55-64$ & $10(22.2 \%)$ & $37(21.8 \%)$ & \\
\hline & $>64$ & $6(13.3 \%)$ & $50(29.4 \%)$ & \\
\hline \multirow[t]{3}{*}{ Marital status, n (\%) } & Single & $11(24.4 \%)$ & $12(7.1 \%)$ & 0.004 \\
\hline & Married & $33(73.3 \%)$ & $152(89.4 \%)$ & \\
\hline & Widowed & $1(2.2 \%)$ & $6(3.5 \%)$ & \\
\hline \multirow[t]{5}{*}{ Occupation, n (\%) } & Student & $2(4.4 \%)$ & $4(2.4 \%)$ & 0.32 \\
\hline & Housewife & $5(11.1 \%)$ & $41(24.1 \%)$ & \\
\hline & Businessperson & $7(15.6 \%)$ & 31 (18.2\%) & \\
\hline & Employed & $10(22.2 \%)$ & $31(18.2 \%)$ & \\
\hline & Peasant & $21(46.7 \%)$ & $63(37.1 \%)$ & \\
\hline \multirow[t]{3}{*}{ Education, n (\%) } & Primary & $28(62.2 \%)$ & $106(62.4 \%)$ & 0.53 \\
\hline & Secondary & $11(24.4 \%)$ & $41(24.1 \%)$ & \\
\hline & Tertiary & $6(13.3 \%)$ & $23(13.5 \%)$ & \\
\hline
\end{tabular}

The overall mean $( \pm \mathrm{SD})$ age of $47.2 \pm 14.5$ years for stroke patients with HIV infection was significantly lower compared to that of $56.1 \pm 15.1$ years among stroke patients who were HIV negative $(P<0.001)$. The mean ages were also significantly different by gender. Male stroke patients with HIV had a mean $( \pm S D)$ age of $46.3 \pm 14.5$ years, while the mean age was $56.6 \pm 14.5$ years among stroke males without HIV infection $(P<0.01)$. The corresponding figures were $47.9 \pm 14.8$ and $55.5 \pm 17.4$ years among female stroke patients with and without HIV infection respectively $(P=0.05)$. The age group with the highest HIV prevalence was that of between 45 and 54 years, while the least HIV prevalence was observed on age group less than 25 years. This however was not statistically significant $(P=0.18)$. 
There were statistically significant differences in the distribution of HIV prevalence by marital status. Eleven of the $45(24.4 \%)$ HIV infected stroke patients were single, whereas only $12 / 170(7.1 \%)$ were singles among the HIV un-infected patients with stroke $(P=0.004)$. A total of 36/45 (80.0\%) HIV infected patients with stroke had their CD4 $4^{+} \mathrm{T}$ - lymphocyte counts (CD4 count) determined. Twenty one (58.3\%) of these had a CD4 count of less than 200 cells/ $\mu \mathrm{l}$. The overall mean $( \pm \mathrm{SD}) \mathrm{CD} 4$ count was $299 \pm 380$ cells/ $\mu \mathrm{l}$; and by sexes was $371 \pm 438$ cells/ $\mu \mathrm{l}$ among males, and $242 \pm 328$ cells $/ \mu 1$ among females $(P$-value $=0.3)$.

\section{Traditional risk factors for stroke}

Among the traditional risk factors for stroke, hypertension was the commonest among both HIV infected and HIV un-infected patients with stroke. Indeed $74.9 \%$ of the 215 patients with stroke had hypertension. However, the proportion of patients with stroke and HIV infection who were hypertensive was 53.3\% (24/45), whereas among those without HIV infection the proportion was $80.6 \%(137 / 170)(P<0.001)$ (Table 2$)$. The proportions of stroke patients who were either obese, diabetic, hypertrglyceridemic or hypercholesterolemic were not significantly different between HIV infected and HIV un-infected patients with stroke.

Table 2: Distribution of the traditional risk factors for stroke by the HIV status of patients admitted with stroke

\begin{tabular}{llll}
\hline Risk Factor & HIV-Positive, $\mathbf{n = 4 5}$ & HIV-Negative, n=170 & P-value \\
\hline Hypertension & $24(53.3 \%)$ & $137(80.6 \%)$ & $<0.001$ \\
Obesity & $6(13.3 \%)$ & $38(22.4 \%)$ & 0.13 \\
Diabetes mellitus & $5(11.1 \%)$ & $32(18.8 \%)$ & 0.16 \\
Hypertriglyceridemia & $7(15.6 \%)$ & $18(10.6 \%)$ & 0.58 \\
Hypercholesterolemia & $9(20.0 \%)$ & $32(18.8 \%)$ & 0.50 \\
\hline
\end{tabular}

\section{Use of anti-retroviral (ARV) drugs}

Of the $45 \mathrm{HIV}$ infected patients with stroke, only 6 (13.3\%) were on ARVs. Of these, four were on Triomune 30 (combination of Stavudine $30 \mathrm{mg}$, Lamivudine $150 \mathrm{mg}$, and Nevirapine 200mg). Two of the 4 had been on Triomune for 4 months, while the other 2 had been on the drug for more than 6 months. The other two patients were on Duovir (combination of Zidovudine $300 \mathrm{mg}$ + Lamivudine $150 \mathrm{mg}$ ) and Efavirenz $600 \mathrm{mg}$ for more than six months. None of the patients was on a Protease inhibitor.

\section{Presenting symptoms and physical findings}

Fever was present in $44.4 \%$ (20/45) of stroke patients with HIV compared to $24.7 \%(42 / 170)$ of stroke subjects who were HIV negative $(P=0.01)$. Furthermore, anaemia was observed in $26.7 \%(12 / 45)$ of stroke patients with HIV infection as compared to $7.6 \%(13 / 170)$ of those who were HIV un-infected $(P=0.001)$. Similarly, the proportions of HIV infected stroke patients having diarrhoea, pulmonary tuberculosis and Kaposi's sarcoma were significantly higher compared to those among HIV-uninfected stroke patients (Table 3). The frequencies of the specific neurological features of stroke such as facial asymmetry, monoplegia, hemiplegia and unconsciousness were not significantly different between the HIV infected and HIV un-infected patients with stroke.

\section{Stroke subtype}

Due to prohibitive cost that had to be borne by the patient or relatives so as to have a CT scan done, only 6 patients had this investigation performed. Consequently, Siriraj scoring 
was adopted. It was found that 75.6\% (34/45) of the HIV infected patients with stroke had an ischaemic stroke subtype. The proportion with ischaemic stroke subtype among the HIVuninfected was $61.8 \%(105 / 170)$. The difference was not statistically significant $(P=0.08)$.

Table 3: Distribution of clinical features by HIV status among patients admitted with stroke

\begin{tabular}{llll}
\hline Clinical feature, $\mathbf{n}(\%)$ & HIV-positive (N= 45) & HIV-negative (N= 170) & $\boldsymbol{P}$-value \\
\hline Fever & $20(44.4 \%)$ & $42(24.7 \%)$ & 0.01 \\
Fits & $2(4.4 \%)$ & $12(7.1 \%)$ & 0.41 \\
Aphasia & $12(26.7 \%)$ & $70(41.2 \%)$ & 0.05 \\
Anaemia & $12(26.7 \%)$ & $13(7.6 \%)$ & 0.001 \\
Numbness & $24(53.3 \%)$ & $73(42.9 \%)$ & 0.14 \\
Headache & $23(51.1 \%)$ & $94(55.3 \%)$ & 0.37 \\
Diarrhoea & $9(20.0 \%)$ & $5(2.9 \%)$ & $<0.001$ \\
Pulmonary tuberculosis & $6(13.3 \%)$ & $2(1.2 \%)$ & 0.001 \\
Kaposi's sarcoma & $3(6.7 \%)$ & $0(0.0 \%)$ & 0.009 \\
Facial asymmetry & $20(44.4 \%)$ & $100(58.8 \%)$ & 0.06 \\
Monoplegia & $5(11.1 \%)$ & $15(8.8 \%)$ & 0.4 \\
Hemiplegia & $40(88.9 \%)$ & $153(91.2 \%)$ & 0.42 \\
Unconsciousness & $7(15.6 \%)$ & $43(25.3 \%)$ & 0.12 \\
\hline
\end{tabular}

\section{Outcome following hospitalization}

The mean $(95 \% \mathrm{CI})$ duration of stay in hospital by stroke patients was found to be 7.1 (6.1, 8.0) days for males; and $8.8(7.6,10.0)$ days for females respectively $(P=0.02)$. However, patients who had stroke and were HIV infected had a longer mean duration of hospital stay of $10.3(8.2,12.5)$ days compared to the mean duration of $7.3(6.5,8.0)$ days among HIV uninfected patients with stroke $(P=0.001)$.

Table 4: Logistic regression analysis of factors associated with mortality among patients with stroke who were HIV infected

\begin{tabular}{lllll}
\hline Analysis & Variable & Odd Ratio & 95\% Conf. Interval & P-value \\
\hline Univariate analysis & Fever & 6.91 & $3.39-14.122$ & 0 \\
& Weight loss & 2.23 & $0.59-8.29$ & 0.23 \\
& Cough & 0.64 & $0.66-5.30$ & 0.48 \\
& Dyspnoea & 2.01 & $0.19-2.21$ & 0.14 \\
& Loss of consciousness & 0.92 & $0.75-3.64$ & 0.25 \\
Multivariate analysis & Anaemia & 2.97 & $1.01-8.77$ & 0.04 \\
& Fever & 5.4 & $1.28-22.95$ & $0.02^{*}$ \\
& Weight loss & 2.1 & $0.36-12.33$ & 0.41 \\
& Anaemia & 1.94 & $0.29-12.86$ & 0.49
\end{tabular}

*Fever was the independent factor associated with mortality

The proportions of stroke patients who had a disability on discharge were $68.9 \%$ (31/45) among those with HIV and 65.9\% (112/170) among those who were HIV un-infected. On the other hand, mortality was $28.9 \%$ (13/45) among stroke patients with HIV, while it was $31.2 \%$ (53/170) among the stroke patients with no HIV. It should be noted though that 
the disability and mortality outcomes following stroke were not statistically different between stroke patients with HIV and those with no HIV.

An attempt was made to determine factors associated with mortality as an outcome on discharge among patients who had stroke. Following logistic regression analysis, the features that were associated with mortality among HIV infected patients with stroke on univariate analysis were the presence of fever and anaemia. However, on multivariate analysis it was only the presence of fever that was independently associated with increased mortality (Table 4).

\section{Discussion}

Stroke has been demonstrated by this study to be a common cause for admission at the Muhimbili National Hospital in Dar es Salaam. A prevalence of $6.8 \%$ is indeed significant, and is actually much higher than what has been reported from Saudi Arabia where the traditional risk factors for stroke such as hypertension, diabetes mellitus, hyperlipidemia and obesity are relatively commoner (Qari, 2000). Another study done in south-western Nigeria found the prevalence to be $3.6 \%$ (Komolafe et al., 2006). Previous studies in Dar es Salaam have also highlighted the fact that stroke is on the increase. Whereas a study done by Kalluvya found that 80 patients had stroke in a period of 1 year (Kalluvya, 1984), a later study by Matuja et al. (1987) reported the number of stroke patients to be 299 over a period of one year among African people in Dar es Salaam. It is therefore imperative that concerted efforts are undertaken to delineate the common causes of stroke in our set up so that appropriate remedial measures are undertaken.

It is unfortunate that relatively younger people get strokes in our area. The mean age of patients with stroke in this study was 54.2 years, whereas in an earlier study the overall mean age was 51 years (Matuja et al., 1987). This contrasts with findings from studies in the developed world whereby about half of stroke cases occur in those aged above 75 years (Bonita et al., 1984). The mean age of 47.2 years for stroke patients with HIV infection found in this study was significantly lower as compared to the mean age among patients with stroke who were not HIV infected. This was also true with the mean ages by gender in keeping with other studies (Hoffmann et al., 2000; Imam, 2002; Kumwenda et al., 2005; Ortiz et al. 2007; Tipping et al., 2007). This fact adds weight to the possible influence of HIV on stroke causation. Indeed the age group most affected of 36-55 years is similar to that which is also seriously affected by HIV in Tanzania $(\mathrm{MoH}, 2005)$. Similar findings have been reported among patients with stroke in black South African HIV positive patients (Mochan et al. 2005).

The impact of HIV appears important as deduced from findings of this study. The prevalence of HIV infection among stroke patients has been found to be $20.9 \%$ indicating that the effect of HIV on stroke can not be underestimated. A relatively higher prevalence (48\%) has been reported from Malawi (Kumwenda et al., 2005).

It would appear from findings of this study that the ischaemic type of stroke is the predominant stroke sub-type. Based on Siriraj scoring, about $75.6 \%$ of HIV-infected stroke patients had an ischemic stroke sub-type, whereas among those who were HIV negative $61.8 \%$ of them had ischaemic type. This is in keeping with another study which also showed ischaemic type of stroke to be the common sub-type irrespective of the HIV serostatus (Kumwenda et al., 2005). 
It was observed that majority of the patients with stroke and HIV infection had severe immunodeficiency. Over half of them had CD4 counts of less than 200 cells/ $\mu 1$. A similar finding of a higher number of patients having low CD4 counts in patients presenting with stroke was observed in a study done in South Africa (Ortiz et al., 2007). It is indeed known that a CD 4 count of less than 200 cells/ $\mu$ is associated with a higher risk for stroke (Cole et al., 2004). Furthermore, carotid intima was found to be thicker in HIV infected patients and rapid progression was more likely in those with immunodeficiency (Hsue et al., 2004). Stroke could therefore be a late manifestation of HIV infection, and hence early detection of the infection is of importance.

From this study, the contribution of ARV use in stroke causation is of very limited influence, if any, as majority of the patients had not been on long-term ARV use, and indeed none was on a protease inhibitor. It would therefore appear that other possible theories play some roles. These include cardiac embolism secondary to cardiomyopathy, meningitis (Cosnett, 1969; Bahemuka, 1979; Kumwenda et al., 2005), non-bacterial thrombotic endocarditis and opportunistic central nervous infections like toxoplasmosis, cryptococcal meningitis and tuberculosis, as well as elevated systemic inflammation leading to atherosclerosis (Stollberger \& Finsterer, 2002). It could also be through HIV induced vascular damage resulting into a coagulopathy by induction of autoantibodies and protein $S$ deficiency (Mochan et al., 2005). The other possible mechanism could be the occurrence of dyslipidemia. Although reaching no statistical significance, in this study it was found that the proportion of patients with dyslipydemia was higher in the HIV infected group as compared to those without HIV infection. The mechanism contributing to these lipid abnormalities remains unknown, and indeed they could occur independently from use of ARV therapy (Riddler et al., 2003). Grunfeld et al. (1991) found that HIV infection was associated with elevated triglyceride levels that worsened with progression of HIV-related disease.

The clinical picture of patients with stroke and HIV infection is of importance. As noted in this study, they are younger, and tend to have other HIV associated manifestations such as fever, anaemia, diarrhoea and tuberculosis. This has been reported earlier (Tipping et al., 2007) (Azzimondi et al., 1995). It follows that the presence of such conditions in a patient with stroke should alert clinicians for the possibility of HIV.

As noted in another study (Saxena et al., 2007), clinicians should also be aware of the finding from this study of a longer mean duration of hospitalization among stroke patients with HIV compared to those who are HIV un-infected. They should therefore aggressively manage the other concomitant illnesses especially those presenting with fever which has been found to be associated with poor outcome.

Received 17 December 2009

Revised 26 January 2010

Accepted 27 January 2010

\section{References}

Azzimondi, G., Bassein, L., Nonino, F., Fiorani, L. Vignatelli, L., Re, G. D'Alessandro, R. (1995) Fever in acute stroke worsens prognosis. A prospective study. Stroke 26, 20402043. 
Bahemuka, M. (1979) Cerebrovascular accidents (strokes) in young normotensive Africans: a preliminary report of a prospective survey. East African Medical Journal 56, 661-664.

Bonita, R., Beaglehole, R. \& North, J.D. (1984) Event, incidence and case fatality rates of cerebrovascular disease in Auckland, New Zealand. American Journal of Epidemiology 120, 236-243.

Brew, B.J. (1993) Transient ischaemic attacks (TIA's) in HIV-1 infection. International Conference on AIDS. 9.

Brown, T.T., Cole, S.R., Li, X., Kingsley, L.A., Palella, F.J., Riddler, S.A. Visscher, B.R., Margolick, J.B. \& Dobs, A.S. (2005) Antiretroviral therapy and the prevalence and incidence of diabetes mellitus in the multicenter AIDS cohort study. Archives of Internal Medicine 165, 1179-1184.

Cole, J.W., Pinto, A.N., Hebel, J.R., Buchholz, D.W., Earley, C.J. \& Johnson, C.J. (2004) Acquired immunodeficiency syndrome and the risk of stroke. Stroke 35, 51-56.

Connor, M.D., Thorogood, M., Casserly, B., Dobson, C., Warlow, C.P. \& SASPI Team (2004). Prevalence of stroke survivors in rural South Africa: results from the Southern Africa Stroke Prevention Initiative (SASPI) Agincourt field site. Stroke 35, 627-632.

Cosnett, J.E. (1969) Strokes in young people: experience in a Bantu hospital. South African Medical Journal 43, 501-507.

Engstrom, J.W., Lowenstein, D.H. \& Bredesen, D.E. (1989) Cerebral infarctions and transient neurologic deficits associated with acquired immunodeficiency syndrome. American Journal of Medicine 86, 528-532.

Erem, C., Arslan, C., Hacihasanoglu, A., Deger, O., Topbas, M., Ukinc, K., Ersoz, H. O. \& Telatar, M. (2004) Prevalence of obesity and associated risk factors in a Turkish population (Trabzon city, Turkey). Obesity Research 12, 1117-1127.

Evers, S., Nabavi, D., Rahmann, A., Heese, C., Reichelt, D. \& Husstedt, I. W. (2003) Ischaemic cerebrovascular events in HIV infection: a cohort study. Cerebrovascular Diseases 15, 199-205.

Grunfeld, C., Kotler, D.P., Shigenaga, J.K., Doerrler, W., Tierney, A., Wang, J., Pierson, R.N., Jr., \& Feingold, K.R.. (1991) Circulating interferon-alpha levels and hypertriglyceridemia in the acquired immunodeficiency syndrome. American Journal of Medicine 90, 154-162.

Hoffmann, M., Berger, J.R., Nath, A. \& Rayens, M. (2000) Cerebrovascular disease in young, HIV-infected, black Africans in the KwaZulu Natal Province of South Africa. Journal of Neurovirology 6, 229-336.

Hsue, P.Y., Lo, J.C., Franklin, A., Bolger, A.F., Martin, J.N., Deeks, S.G., Waters, D.D. (2004) Progression of atherosclerosis as assessed by carotid intima-media thickness in patients with HIV infection. Circulation 109, 1603-1608.

Imam, I. (2002) Stroke: a review with an African perspective. Annals of Tropical Medicine and Parasitology 96, 435-445.

Jones, M.R., Horner, R.D., Edwards, L.J., Hoff, J., Armstrong, S.B., Smith-Hammond, C.A., Matchar, D.B. \& Oddone, E.Z. (2000) Racial variation in initial stroke severity. Stroke 31, 563-567.

Joubert, J. (1991) The MEDUNSA Stroke Data Bank. An analysis of 304 patients seen between 1986 and 1987. South African Medical Journal 80, 567-570.

Kalluvya, S.E. (1984) Stroke in the African at Muhimbili Medical Centre Internal Medicine. MMed Dissertation. University of Dar es Salaam, Tanzania. 
Kasper, D.L., Fauce, A.S., Longo, D.L., Braunwald, E., Hauser, S.L. \& Jameson, J.L. (2005) Harrison's Principles of Internal Medicine. 16th ed. New York: McGraw Hill.2005

Komolafe, M.A., Ogunlade, O. \& Komolafe, E.O. (2006) Stroke mortality in a teaching hospital in south western Nigeria. African Neurological Sciences 25, 75-77.

Kumwenda, J.J., Mateyu, G., Kampondeni, S., van Dam, A.P., van Lieshout, L. \& Zijlstra, E.E. (2005) Differential diagnosis of stroke in a setting of high HIV prevalence in Blantyre, Malawi. Stroke 36, 960-964.

Matuja, W., Janabi, M., Kazema, R., Mashuke, D. (2004) Stroke subtypes in Black Tanzanians: a retrospective study of computerized tomography scan diagnoses at Muhimbili National Hospital, Dar es Salaam. Tropical Doctor 34, 144-146.

Matuja, W. B. P. (1987) Stroke among African people in Dar es Salaam, Tanzania. MEDICOM 9, 7-9.

Mochan, A., Modi, M. \& Modi, G. (2005) Protein S deficiency in HIV associated ischaemic stroke: an epiphenomenon of HIV infection. Journal of Neurology, Neurosurgery and Psychiatry 76, 1455-1456.

Mochan, A., Modi, M. \& Modi, G. (2003) Stroke in black South African HIV-positive patients: a prospective analysis. Stroke 34, 10-15.

Murphy, R.L., Brun, S., Hicks, C., Eron, J.J., Gulick, R., King, M., White, A.C., Jr., Benson, C., Thompson, M., Kessler, H.A., Hammer, S., Bertz, R. \& Hsu, A. (2001) ABT378/ritonavir plus stavudine and lamivudine for the treatment of antiretroviral-naive adults with HIV-1 infection: 48-week results. AIDS 15, F1-9.

Murray, C.J. \& Lopez, A.D. (1997) Alternative projections of mortality and disability by cause 1990-2020: Global Burden of Disease Study. Lancet 349 (9064), 1498-1504.

$\mathrm{MoH}$ (2005) HIV/AIDS/STI Surveillance Report, 20, 1-9. National AIDS Control Programme, Ministry of Health, Tanzania

Nicholas, A.B., Nicki, R.C, Brian, R.W. \& John, A.A.H., (2006) Davidson's Principles \& Practice of Medicine.

Nyandaiti, Y.W. \& Bwala, S.A. (2008) Validation study of the Siriraj stroke score in Northeast Nigeria. Nigerian Journal of Clinical Practice 11, 176-180.

Ortiz, G., Koch, S., Romano, J.G., Forteza, A.M. \& Rabinstein, A. A. (2007) Mechanisms of ischemic stroke in HIV-infected patients. Neurology 68, 1257-1261.

Poungvarin, N., Viriyavejakul, A. \& Komontri, C. (1991) Siriraj stroke score and validation study to distinguish supratentorial intracerebral haemorrhage from infarction. BMJ 302(6792), 1565-1567.

Qari, F. A. (2000) Profile of stroke in a teaching university hospital in the western region. Saudi Medical Journal 21, 1030-1033.

Qureshi, A.I., Janssen, R.S., Karon, J.M., Weissman, J.P., Akbar, M.S., Safdar, K. \& Frankel, M.R. (1997) Human immunodeficiency virus infection and stroke in young patients. Archives of Neurology 54, 1150-1153.

Riddler, S.A., Smit, E., Cole, S.R., Li, R., Chmiel, J.S., Dobs, A., Palella, F., Visscher, B., Evans, R. \& Kingsley, L.A. (2003) Impact of HIV infection and HAART on serum lipids in men. Journal of American Medical Association 289, 2978-2982.

Saxena, S.K., Koh, G.C., Ng, T.P., Fong, N.P. \& Yong, D. (2007) Determinants of length of stay during post-stroke rehabilitation in community hospitals. Singapore Medical Journal 48, 400-407. 
Stollberger, C. \& Finsterer, J. (2002) Role of infectious and immune factors in coronary and cerebrovascular arteriosclerosis. Clinical and Diagnostic Laboratory Immunology 9, 207215.

Stuart-Shor, E. M., Wellenius, G. A., Dellolacono, D. M. \& Mittleman, M. A. (2009) Gender differences in presenting and prodromal stroke symptoms. Stroke 40, 1121-1126.

TACAIDS (2008) Tanzania HIV/AIDS and Malaria Indicator Survey 2007-08. Tanzania Commission for AIDS.

Tipping, B., de Villiers, L., Wainwright, H., Candy, S. \& Bryer, A. (2007) Stroke in patients with human immunodeficiency virus infection. Journal of Neurology, Neurosurgery and Psychiatry 78, 1320-1324.

Walker, R.W., McLarty, D.G., Kitange, H.M., Whiting, D., Masuki, G., Mtasiwa, D.M., Machibya, H., Unwin, N. \& Alberti, K.G. (2000) Stroke mortality in urban and rural Tanzania. Adult Morbidity and Mortality Project. Lancet 355(9216), 1684-1687.

Wiredu, E.K. \& Nyame, P.K. (2001) Stroke-related mortality at Korle Bu Teaching Hospital, Accra, Ghana. East African Medical Journal 78, 180-184.

Yusuf, S., Hawken, S., Ounpuu, S., Dans, T., Avezum, A., Lanas, F. McQueen, M., Budaj, A., Pais, P., Varigos, J. \& Lisheng, L. (2004) Effect of potentially modifiable risk factors associated with myocardial infarction in 52 countries (the INTERHEART study): case-control study. Lancet 364 (9438), 937-952. 